\title{
P Segregation to Low-Angle D-Fe Grain Boundaries
}

\author{
Chunfei Li and David B. Williams
}

\section{Department of Materials Science and Engineering, Lehigh University, Bethlehem, PA 18015}

The amount of trace element segregation to individual grain boundaries can be quite variable [e.g., 1-2]. The first step to describe boundary structure is to specify the misorientation of grains across a boundary, which is usually denoted by the angle/axis pair. Focusing on the misorientation angle, grain boundaries are classified in terms of low and high angle boundaries, where the angles of misorientation are less or more than $15^{\circ}$, respectively. Alternatively, boundaries with certain discrete angle/axis pairs, where, if the lattices of the two grains are allowed to interpenetrate a fraction of the lattice sites coincide, are called Coincidence Site Lattice (CSL) boundaries. The reciprocal of the density of CSL points is represented by $\sum$. Since lower angle and/or low $\sum$ boundaries would have less free volume, it is reasonable to expect less segregation to such boundaries and there are reports indicating that the segregation to low $\sum$ grain boundary was reduced. By using Analytical Electron Microscopy (AEM), Keast and Williams [1] reported the segregation of $\mathrm{Bi}$ to a $\Sigma 9 \mathrm{Cu}$ grain boundary while the neighboring $\sum 3$ grain boundary sees no Bi segregation. This is in accordance with the concept that a boundary with low $\sum$ value or a low angle of misorientation implies a good fit of adjacent grains and the driving force for segregation is weak. The misorientation angle for CSL grain boundaries with $\sum$ value up to 9 is larger than $15^{\circ}$, implying that small angle and low $\sum$ grain boundaries are two independent special types under the present definition. Suzuki et al. [3] reported $\mathrm{P}$ segregation to a low-angle grain boundary in Fe by using Auger Electron Spectroscopy (AES), where the segregation level was on the same order as for other 28 high angle boundaries.

In the present work, we report $\mathrm{P}$ segregation to individual grain boundaries in a rapidly solidified Fe$0.6 \mathrm{wt} \% \mathrm{P}$ alloy. Grain boundary chemical analysis was performed by X-ray Mapping (XRM), which was carried out in HB 603 Scanning Transmission Electron Microscope (STEM). The results of elemental mapping were converted to P concentration image by the Cliff-Lorimer [4] method. The crystallographic analysis of grain boundaries was performed on a Philips EM 420 TEM by using ACT [5-6] (Automatic Crystallography for TEM (Transmission Electron Microscopy)) and CBED (Convergent Beam Electron Diffraction).

In the present work 25 boundaries were checked by XRM. It was found that the segregation to individual grain boundary is indeed anisotropic. Examples of boundaries with low ((a) and (b)) and high ((c) and (d)) P concentrations are shown in Fig. 1. P concentration on the boundary shown in Fig. 1(b) is lower than that in Fig. 1(d). The angle/axis pair for the grain boundary shown in Fig. 1(a)

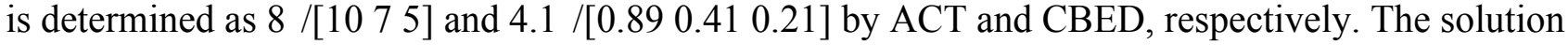
for the boundary shown in Fig. 1(c) is $45^{\circ} /\left[\begin{array}{lll}8 & 2 & 1\end{array}\right]$ and $46.4^{\circ} /\left[\begin{array}{lll}0.95 & 0.22 & 0.21\end{array}\right]$. Then, the boundary in Fig. 1(a) is a small angle one. It is confirmed that the angle/axis pair for the boundary shown in Fig. 1(c) does not correspond to that for CSL boundaries with $\sum$ value up to 9, implying that this boundary is high angle and non-low- $\sum$ type. Combination of the chemical and crystallographic analysis results reveals that the amount of $\mathrm{P}$ segregation to a small angle grain boundary is less. In addition, advantages of AEM in grain boundary segregation study will be discussed and results of misorientation determined from ACT and CBED will be compared with each other. 


\section{References}

[1] V.J. Keast and D.B. Williams, Acta Mater. 47 (1999) 3999.

[2] T. Ogura, C.J. McMahon, H.C. Feng and V. Vitek, Acta Metall. 26 (1978) 1317.

[3] S. Suzuki, K. Abiko and H. Kimura, Scripta Metall. 15 (1981) 1139.

[4] G. Cliff and G.W. Lorimer, J. Microsc. 103 (1975) 203.

[5] S.I. Wright and D.J. Dingley, Mater. Sci. Forum 273-275 (1998) 209.

[6] C. Li and D.B. Williams, Micron (submitted).
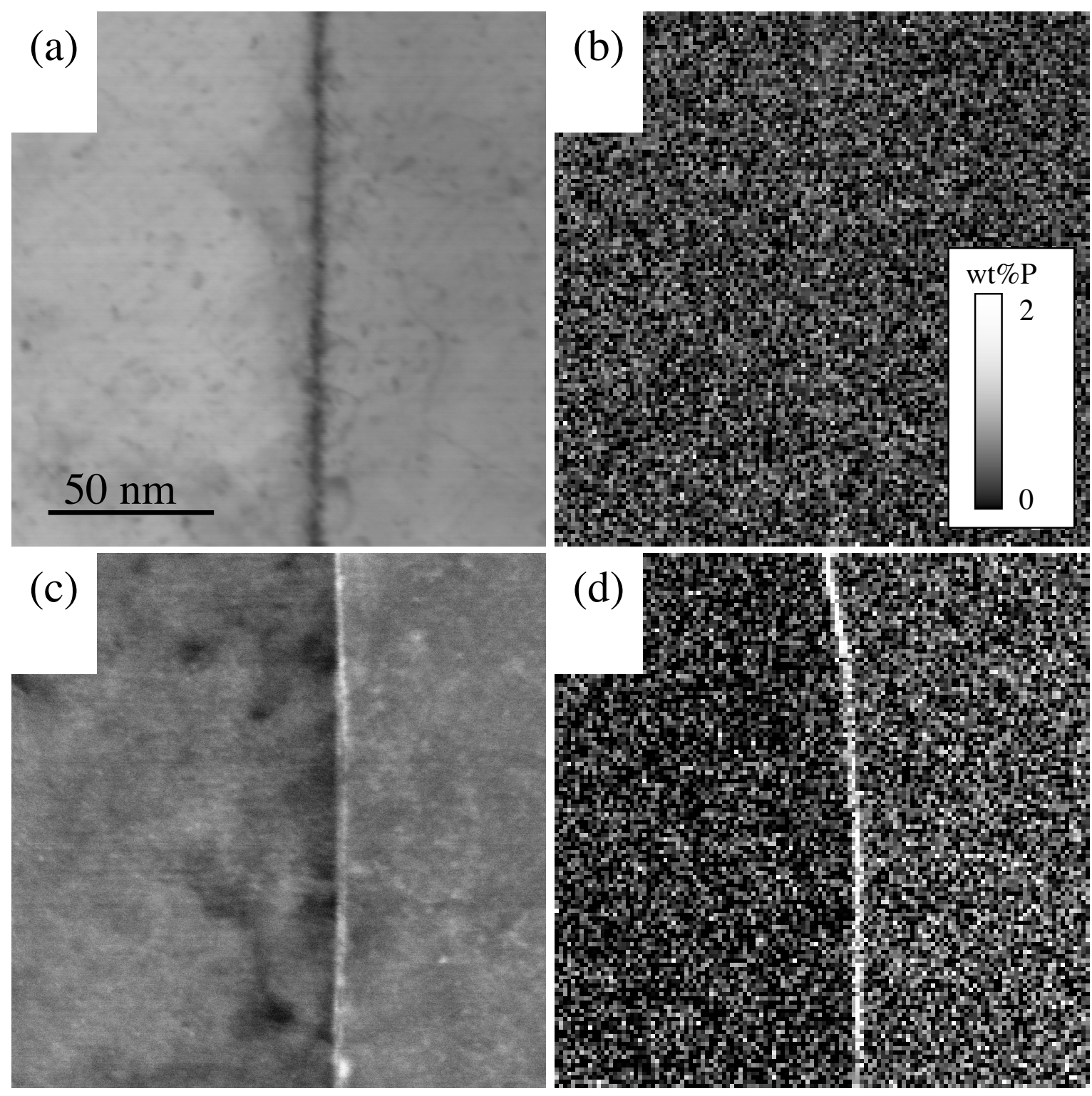

Fig. 1 Bright-field and P concentration images for boundaries with low: (a) and (b), and high: (c) and (d) P concentrations, respectively. In term of angle/axis pair, the misorientations between the adjacent grains in (a) and (c) are $4.1 \%$ [ 0.890 .410 .21$]$ and $46.4^{\circ} /[0.950 .220 .21]$, respectively, which were determined by CBED. 